\title{
A CRISPR view of the 2020 Nobel Prize in Chemistry
}

\author{
Katherine E. Uyhazi and Jean Bennett \\ Center for Advanced Retinal and Ocular Therapeutics (CAROT), Department of Ophthalmology, Scheie Eye Institute, Department of Cell and Developmental Biology, University of Pennsylvania Perelman \\ School of Medicine, Philadelphia, Pennsylvania, USA.
}

$\mathbf{T}_{\text {he }}$ he 2020 Nobel Prize in Chemistry was awarded to Emmanuelle Charpentier of the Max Planck Unit for the Science of Pathogens and Jennifer Doudna of the University of California, Berkeley, for their discovery of the CRISPR/Cas9 genetic scissors that have revolutionized genome editing (1).

While studying Streptococcus pyogenes, Charpentier discovered that a molecule called tracrRNA was part of bacteria's ancient immune system, CRISPR/Cas, that cleaves viral DNA (2). Meanwhile, Doudna was deciphering the structure and biologic function of RNA enzymes and recognized the RNA-based surveillance system in bacteria (3). When they met at a scientific meeting in 2011, Charpentier and Doudna had a eureka moment, realizing that jointly, they could harness the RNAbased complex to develop a novel gene editing system. Through a series of experiments published in 2012 (4), they were the first to define an in vitro CRISPR/Cas9 system that not only could cut viral DNA but also could be programmed with an engineered guide RNA to cut any DNA at a sequence-specific site (Figure 1). Just as the discovery of restriction enzymes in the 1960s paved the way to an era of recombinant DNA technology, the CRISPR/Cas9 discoveries of Charpentier and Doudna have revolutionized our genome editing toolbox. In less than a decade, the applications of this powerful technology have exploded worldwide and have already led to advances in plant and animal engineering, personalized medicine, and promising clinical therapeutics.

\section{From the CRISPR drawer to the clinic}

While the potential and wide-ranging applications of CRISPR/Cas continue to expand,
CRISPR has already been at work in our dairy products, as the antiviral defense mechanisms maintain the consistency and acidity of yogurt cultures. In the agriculture industry, studies are underway to harness CRISPR to engineer a wide variety of plants (5) to have better yields and superior resistance to pests and environmental stresses. Whether consumers will adapt to CRISPRmodified crops remains to be seen, although there may be a lower threshold for acceptance of genetically engineered medications. Cannabis represents one such potentially lucrative target (6) because engineering could allow more efficient extraction from single-cell organisms engineered to secrete the cannabinoids into culture medium. CRISPR is also being evaluated for its potential to engineer livestock to eliminate the risk of deadly infections and to boost growth as well as to generate humanized organs that ultimately could safely be used for xenotransplantation.

Meanwhile, diagnostic applications of CRISPR have emerged and continue to expand (7). CRISPR proteins can be designed as biosensors to identify a specific DNA or RNA target sequence. The assays are ultrasensitive, low cost, and fast. CRISPR has been used to identify various pathogens in clinical samples, including Zika virus, human papillomavirus (HPV), tuberculosis, enterovirus, pertussis, and malaria (8). Perhaps one of the timeliest applications is an FDA-approved test for SARS-CoV-2 infection.

Although the first CRISPR/Cas clinical trial was initiated only four years ago, there are currently 28 CRISPR/Cas studies listed on ClinicalTrials.gov that are recruiting subjects. Although some of these studies are diagnostic or explore basic biologic questions, 21 aim to develop new thera-
Conflict of interest: JB has served on advisory boards for GenSight Biologics; SparingVision; Akouos, Inc; Life Biosciences; and Odylia Therapeutics and has consulted for Spark Therapeutics. She served as the scientific director for clinical trials run by Spark Therapeutics.

Copyright: () 2021, American Society for Clinical Investigation.

Reference information: J Clin Invest. 2021;131(1):e145214. https://doi.org/10.1172/JCI145214. peutics. The vast majority of the therapeutic studies use ex vivo strategies whereby CRISPR-modified cells are infused to treat malignancies or lethal hematologic disorders (Figure 1). For example, studies of CTX001, produced by CRISPR Therapeutics, aim to edit the BCL11A gene in order to increase the production of fetal hemoglobin as a potential treatment for $\beta$-thalassemia and sickle cell disease (8). CRISPR-based strategies are also being developed to treat mesothelin-positive solid tumors, hematologic malignancies (leukemia, lymphoma, multiple myeloma, Epstein-Barr virusrelated cancer), renal cell carcinoma, and metastatic gastrointestinal cancers $(8,9)$.

There are four studies in progress that involve direct, in vivo delivery of CRISPR editing systems (Figure 1) (8). One study targets HPV-related cervical neoplasia and involves repeated topical applications of a CRISPR/Cas9 plasmid targeting HPV (8). The other three studies involve a one-time in vivo intervention. The first involves intravenous delivery of the gene editing system mixed with lipid nanoparticles in subjects with hereditary transthyretin amyloidosis (8). The other two studies target the eye, and because of the accessibility of this tissue, delivery is achieved under direction visualization (8). A study of refractory herpetic keratitis involves corneal injection. The second ocular study addresses a hereditary congenital blindness, LCA10, caused by a common mutation in the CEP290 gene that leads to creation of a premature stop codon and maldevelopment and malfunction of photoreceptor cells. The LCA10 study is the only CRISPR study to date to employ subretinal delivery of the gene editing material through an adeno-associated viral vector (AAV5), which targets the primary disease-causing cells efficiently.

\section{Safety and ethical considerations}

There are a number of safety concerns relating to in vivo delivery of gene editing 
A

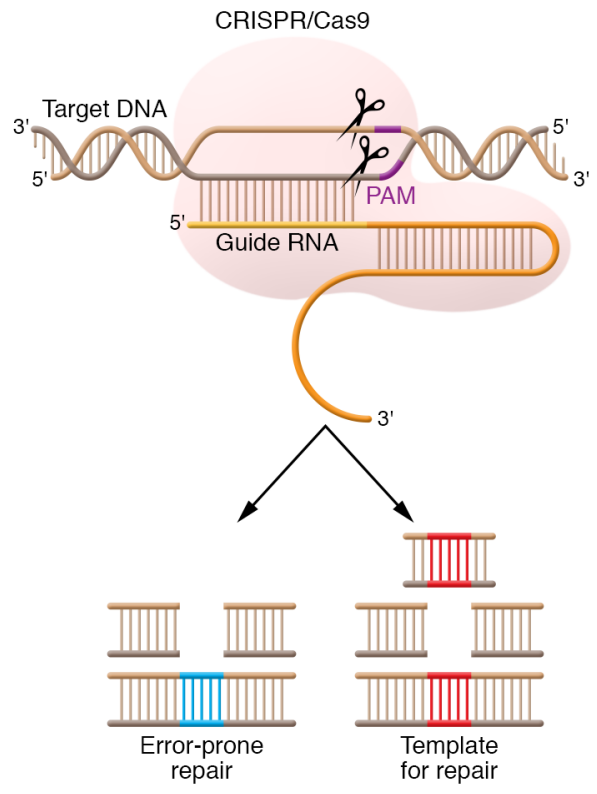

B

Figure 1. Programmable molecular scissors: from concept to clinical applications. (A) A guide RNA recognizes a genomic sequence, and Cas9 cuts the DNA upstream of the protospacer adjacent motif (PAM) sequence. Genome editing can lead to error-prone repair (small insertions or deletions following nonhomologous end joining that usually lead to lack of expression) or to defined changes (using a template for homology-directed repair). Additional strategies can be incorporated to precisely modify specific nucleotides or to target RNA (not shown). (B) Several clinical applications for CRISPR/Cas exist or are in development. In vivo application generally requires incorporation of the editing reagents in a vector (either nonviral such as a gel or in lipid nanoparticles or recombinant virus such as adeno-associated virus, AAV). In vivo trials are ongoing to treat blindness (specifically herpes simplex virus keratitis or Leber's congenital amaurosis) as well as HPV-driven cervical neoplasia and transthyretin amyloidosis, a rare congenital disorder that affects multiple organs due to amyloid deposits. Ex vivo applications include diagnostic applications, such as for SARS-CoV-2 infection, as well as clinical therapies based on CRISPR/Cas modification of cells, which are then used to treat patients. Cell-based therapies to date include applications in hematologic disorders and malignancies, renal cell carcinoma, and metastatic gastrointestinal cancers.

reagents, one of which relates to the potential of a harmful immune response (8). Because the Cas protein is derived from bacteria, it is likely highly immunogenic. In fact, in vitro-transcribed guide RNA has been shown to trigger innate immune responses, and anti-Cas9 antibodies have been identified in human serum. These preexisting antibodies could neutralize the test reagent, thereby preventing any benefit, or worse, could lead to inflammation and tissue destruction. In addition, vectors used to encapsulate and deliver the editing reagents may incite an inflammatory response, which in terminally differentiated tissues, such as the retina, could lead to permanent tissue damage. However, the relatively immunoprivileged environment of the retina (including tissue planes that limit drug distribution, a lack of a direct blood supply, and presence of immune suppressive molecules) was likely beneficial in the development of Luxturna, an AAV2-mediated gene augmentation therapy for congenital blindness that the FDA approved in 2017. Inherited retinal diseases thus represent a promising target for CRISPR-based therapies, with several studies already in the pipeline.

Besides immune response, another safety concern of in vivo delivery of CRISPR/Cas is the potential for off-target effects (8). Careful selection of guide sequences, incorporation of cell-specific promoters, and transient delivery of Cas 9 may minimize these risks. The greatest concern is that a malignancy could result. By selecting tissue targets that can be directly visualized (the cervix, cornea, retina) in the first sets of in vivo CRISPR/Cas studies, regular noninvasive safety screening can be achieved. Finally, ethical concerns have already attracted the world's attention, when CRISPR-edited human embryos were implanted and twins were ultimately born in China (10). Doudna was, in fact, the first person in the United States to hear about this work, when she received an email from the Chinese scientist planning the birth announcement in 2018 (11). The details of those gene editing procedures and the results are unknown, but the scientist conducting this work earned a three-year jail sentence and a large fine (Rmb3m \$430,000). This incident also stimulated much discussion about the need for international guidelines on human gene editing.

\section{CRISPR patent wars}

Given the wide array of potential applications for CRISPR technology, it is not surprising that intellectual property ownership has attracted much scrutiny (12). Doudna and Charpentier published the first evidence that the CRISPR system could cut targeted bacterial DNA in June 2012. Six months later, Feng Zhang of the Broad Institute and others in Boston showed that CRISPR worked in mammalian cells (13) and were the first to patent the ability to edit human genomes. Both groups (the California- 
Vienna-Charpentier "CVC" group and the Broad/Harvard/MIT group) submitted claims that are currently under litigation by the US Patent Trial and Appeal Board (14). Most recently, the group led by the Broad Institute was granted "priority" for the CRISPR eukaryotic cell patents. However, a patent for one widely used component of the CRISPR toolbox, the single RNA guide, was awarded to CVC. The legal issues are complicated and include priority dates, reduction to practice, and US versus European patent laws.

On top of this, there are accusations that individuals who stand to gain financially have attempted to shape the history of the CRISPR discoveries. It is thus noteworthy that the Nobel Prize selection committee selected Doudna and Charpentier alone as laureates, thus prioritizing discovery over invention and recognizing the inspirational roles of scientists who are women in this important achievement. In fact, the award to Doudna and Charpentier represents the first time two women have shared the Nobel Prize in Chemistry and brings the number of women recipients of this award over the past 120 years to seven $(3.78 \%)$.

\section{Conclusion}

The discoveries by Charpentier and Doudna demonstrate the revolutionary impact that can result from basic research. What once may have seemed an esoteric enzyme of the microbiology world is now a household name that has led to simple, efficient, and cost-effective genome editing and groundbreaking advances in biol- ogy and medicine. This Nobel Prize also reminds us of the importance of interdisciplinary scientific collaboration, of eureka moments made possible by human interaction, and of the power of scientific curiosity and ingenuity. While CRISPR-based genome editing has the potential to treat (or even cure) inherited diseases, the power of rewriting the genome has also raised concerns about what is morally acceptable. The future of this exciting field therefore demands a careful balance of scientific feasibility and ethical vigilance.

\section{Acknowledgments}

JB is supported by a Sponsored Research Agreement from Gyroscope, Inc; the Brenda and Matthew Shapiro Stewardship; and the Robert and Susan Heidenberg Investigative Research Fund for Ocular Gene Therapy. KEU is supported by the National Eye Institute of the National Institutes of Health (K08EY031754). JB and KEU are supported by CAROT, Scheie Eye Institute, the Paul and Evanina Bell Mackall Foundation Trust, the National Foundation for Cancer Research, and Research to Prevent Blindness.

Address correspondence to: Jean Bennett, 309C Stellar-Chance Labs, 422 Curie Blvd, Philadelphia, Pennsylvania 19104, USA. Phone: 215.898.0915; Email: jebennet@ pennmedicine.upenn.edu.

1. Nobel Prize. Press release: the Nobel Prize in Chemistry 2020. Nobel Prize website. https:// www.nobelprize.org/prizes/chemistry/2020/ press-release/. October 7, 2020. Accessed November 16, 2020.
2. Deltcheva E, et al. CRISPR RNA maturation by trans-encoded small RNA and host factor RNase III. Nature. 2011;471(7340):602-607.

3. Wiedenheft B, et al. Structures of the RNA-guided surveillance complex from a bacterial immune system. Nature. 2011;477(7365):486-490.

4. Jinek M, et al. A programmable dual-RNAguided DNA endonuclease in adaptive bacterial immunity. Science. 2012;337(6096):816-21.

5. Liu X, et al. Application of CRISPR/Cas9 in plant biology. Acta Pharm Sin B. 2017;7(3):292-302.

6. Dolgin E. The bioengineeering of cannabis. Nature. 2019;572:S5-S7.

7. Maxmen A. Faster, better, chaper: the rise of CRISPR in disease detection. Nature. 2019;566(7745):437.

8. Hirakawa MP, et al. Gene editing and CRISPR in the clinic: current and future perspectives. Biosci Rep. 2020;40(4):BSR20200127.

9. Ernst MPT, et al. Ready for repair? Gene editing enters the clinic for the treatment of human disease. Mol Ther Methods Clin Dev. 2020;18:532-557.

10. Greely HT. CRISPR'd babies: human germline genome editing in the 'He Jiankui affair'. J Law Biosci. 2019;6(1):111-183.

11. Kuckler H. Jennifer Doudna, Crispr scientist, on the ethics of editing humans. Financial Times. https://www.ft.com/content/6d063e48-435911ea-abea-0c7a29cd66fe. January 31, 2020. Accessed November 16, 2020.

12. Reader R. Patent Nobel-winning CRISPR scientists are fighting for its patent. Fast Company. https://www.fastcompany.com/90561762/ nobel-prize-jennifer-doudna-emmanuellecharpentier-crispr-patent-lawsuit. October 10, 2020. Accessed November 16, 2020.

13. Cong L, et al. Multiplex genome engineering using CRISPR/Cas systems. Science. 2013;339(6121):819-823.

14. Cohen J. The latest round in the CRISPR patent battle has an apparent victor, but the fight continues. Science. https://www.sciencemag.org/ news/2020/09/latest-round-crispr-patentbattle-has-apparent-victor-fight-continues. September 11, 2020. Accessed November 16, 2020. 